\title{
ANALYSIS OF THE IMPULSIVE PHASE OF A SOLAR FLARE AT SUBMILLIMETER WAVELENGTHS
}

\author{
JEAN PIERRE RAULIN ${ }^{1}$, VLADIMIR S. MAKHMUTOV ${ }^{1, *}$, \\ PIERRE KAUFMANN $^{1, \dagger}$, ALESSANDRA ABE PACINI $^{1, \ddagger}$, THOMAS LÜTHI ${ }^{2}$, \\ HUGH S. HUDSON ${ }^{3}$ and DALE E. GARY ${ }^{4}$ \\ ${ }^{1}$ CRAAM, Universidade Presbiteriana MacKenzie, São Paulo, Brasil \\ (e-mail: raulin@craam.mackenzie.br) \\ ${ }^{2}$ IAP, University of Bern, Bern, Switzerland \\ ${ }^{3}$ SSL, University of California at Berkeley, CA, U.S.A. \\ ${ }^{4}$ CSTR, New Jersey Institute of Technology, Newark, U.S.A.
}

(Received 30 April 2004; accepted 5 July 2004)

\begin{abstract}
We present a report on the strong X5.3 solar flare which occurred on 25 August 2001, producing high-level $\gamma$-ray activity, nuclear lines and a dramatic long-duration white-light continuum. The bulk of millimeter radio fluxes reached a peak of $\sim 100000$ solar flux units at $89.4 \mathrm{GHz}$, and a few thousands of solar flux units were detected in the submillimeter range during the impulsive phase. In this paper we focus on and discuss (i) the implications inferred from high frequency radio observations during the impulsive phase; (ii) the dynamics of the low corona active region during the impulsive phase. In particular we found that $4-5 \times 10^{36}$ accelerated $(>20 \mathrm{keV})$ electrons s $\mathrm{s}^{-1}$ radiating in a $1000-1100 \mathrm{G}$ region, are needed to explain the millimeter to submillimeter-wave emissions. We present evidence that the magnetic field in the active region was very dynamic, and that strong non-thermal processes were triggered by the appearance of new, compact, low-lying (few thousand kilometers) loop systems, suggesting the acceleration site(s) were also located in the low solar atmosphere.
\end{abstract}

\section{Introduction}

Improved understanding of solar flare physics can be achieved using highfrequency radio observations. The reason is that an important fraction of the energy released during solar events is transferred to ambient electrons, which gain energy up to few tens of $\mathrm{MeV}$ or more. These particles should in turn radiate a substantial amount of short radio waves, down to millimeter and submillimeter domains (Dulk, 1985; White et al., 1992; Ramaty et al., 1994), when they propagate along coronal magnetic fields. Some of these particles can precipitate in the direction of the solar surface and produce hard X-ray $/ \gamma$-ray emission through the non-thermal bremsstrahlung mechanism. The hard X-ray $/ \gamma$-ray emission provides diagnostics of the same energetic electrons, but the radiation depends differently on the local

\footnotetext{
*Also Lebedev Physical Institute, Moscow, Russia.

${ }^{\dagger}$ Also part-time at CSS, Universidade Estadual de Campinas, Campinas, Brasil.

¥Also Instituto Nacional de Pesquisas Espaciais, São José dos Campos, Brasil.
} 
physical parameters; their comparison thus can bring a wealth of information on the transport of the accelerated particles (Ramaty et al., 1994). Similar progress would be expected if we were to have better knowledge of the relationships between sites of radiation and acceleration. While the former are easily observed, the latter are indirectly inferred. The acceleration site is sometimes inferred to be at an altitude range of 5600-17000 km (Aschwanden et al., 1996) consistent with the cusp region above the flaring loop or in coronal regions where the ambient electronic density is $0.6-10 \times 10^{9} \mathrm{~cm}^{-3}$ (Aschwanden and Benz, 1997). On the other hand, more recently, acceleration regions have been described as the interaction sites between magnetic structures (Hanaoka, 1999), sometimes involving compact loop systems (Grechnev and Nakajima, 2002) but always suggesting strong magnetic field changes in the main flare site, as in Kundu et al. (2001).

Solar high-frequency ( $\mathrm{mm}$ and submm) radio observations are important, since they reveal the presence of electrons with energies of a few $\mathrm{MeV}$ rather than those of a few hundred $\mathrm{keV}$, which produce the hard X-ray emissions. This is because the gyrofrequency $\left(v_{B}\right)$ in the solar corona does not exceed a few $\mathrm{GHz}$, and therefore $405 \mathrm{GHz}$ synchrotron emission is only possible at harmonics above 100. Since the synchrotron intensity of a particle with a Lorentz factor $\gamma$ is $\sim \gamma^{3} \times v_{B}$, we see that only relativistic/ultra-relativistic electrons will radiate in the submillimeter wavelength range. Such observations then constitute important tests for the existing acceleration models. The treatment of high frequency radio observations requires the use of full computation codes (like in Ramaty, 1969; Ramaty et al., 1994), since the approximate formulae (Dulk and Marsh, 1982; Dulk, 1985) are only valid for a lower range of harmonic numbers (typically 20-100), and then cannot be used to compute the gyrosynchrotron emission from electrons producing hard X-rays, or synchrotron emission at very high harmonics (Ramaty et al., 1994).

In this paper we present, for the first time, flare observations that include radio waves from 1 to $405 \mathrm{GHz}$, along with hard X-ray imaging observations and $\gamma$-ray time profiles up to a photon energy range of tens of MeV. The submillimeter data were obtained by the new Solar Submillimeter-wave Telescope (SST; Kaufmann et al., 2001a) installed in the Argentinean Andes since May 1999. Numerous aspects of activity detected at 212 and $405 \mathrm{GHz}$, such as fast (100-300 ms) flux density time variations (Kaufmann et al., 2001b), were found, sometimes associated with $\gamma$-ray spikes (Kaufmann et al., 2002). Properties of these fast-time structures are published elsewhere (Raulin et al., 2003; Makhmutov et al., 2003). More recently, a new component was discovered in the teraHertz frequency range (Kaufmann et al., 2004). These results show the importance of the millimeter and submillimeter diagnostics to probe the highest-energy electrons produced during solar flares. In Section 2 we present the instrumental data base used. In Section 3 we discuss the observational results, focusing on the implications of the observed radio spectra when interpreted using synchrotron calculations of Ramaty (1969) and Ramaty et al. (1994), and on the dynamics of the low corona as imaged in soft $\mathrm{X}$-rays. In Section 4 we present our conclusions. 


\section{Observations}

The 25 August 2001 flare occurred at location S17 E34 in the active region NOAA 9591, at around 16:30 UT. It has been classified as optical class 3B, and GOES $(1-8 \AA)$ class X5.3. Intense $\mathrm{H} \alpha$ emission was observed between 16:24 and 19:17 UT (NOAA Solar Geophysical Data Bulletins). The magnetic configuration of the active region was classified as $\beta \gamma \delta$, and it was composed of two large sunspots whose umbrae were located within the same penumbra. White-light images show bright emission starting between 16:26:00 and 16:29:26 UT located along a magnetic neutral line between the two spots, at a site where strong magnetic field gradients were detected on the previous day (24 August). White-light emission decayed after reaching its maximum at about 16:31-16:32 UT. The comparative study of white-light and hard X-ray emissions for the 25 August 2001 flare has been published by Metcalf et al. (2003). In addition, this dramatic white-light flare showed intense $\gamma$-ray emission as well as nuclear lines.

\subsection{INSTRUMENTATION}

The SST observes the Sun at 212 and $405 \mathrm{GHz}$ with six independent receivers (channels 1, 2, 3, 4 at $212 \mathrm{GHz}$, and channels 5, 6 at $405 \mathrm{GHz}$ ), with a time resolution of $1 \mathrm{~ms}$. The multiple-receiver focal array produces a cluster of four beams $(2,3,4,5)$ overlapping at $\sim$ HPBW (4 arc min and 2 arc min at 212 and $405 \mathrm{GHz}$, respectively) projected on the solar disk, while the other two beams $(1,6)$ are projected at a remote $(\sim 7-8$ arc $\mathrm{min})$ site (see for example Figure 1 Kaufmann et al., 2002). This beam disposition allows the estimation of the location of the centroid of emission for a small (compared to HPBW) emitting source, which is used subsequently to compute the source flux density, after correcting the observed antenna temperatures for atmospheric attenuation (determined for the time of the flare as $\sim 0.58$ and 2.6 nepers at 212 and $405 \mathrm{GHz}$, respectively). The calculation of the source flux density also assumes aperture efficiencies of about 0.2 at $212 \mathrm{GHz}$ and 0.13 at $405 \mathrm{GHz}$, obtained from planet observations. The high value of atmospheric opacity for this day means that the error in its determination is the main source of uncertainty ( $25 \%$ and $40 \%$ at 212 and $405 \mathrm{GHz}$, respectively) for the absolute flux density. On 25 August 2001, beams 2, 3, 4, and 5 of the SST were tracking NOAA 9591 where the flare occurred. We used the records of beam 6 , located partially on the solar disk although far away from the active region, to correct the time evolution at $405 \mathrm{GHz}$ from fluctuations of atmospheric origin.

The SST observations were complemented by full-disk measurements obtained at $89.4 \mathrm{GHz}$ by the nulling interferometer at Bern University (Lüthi, 2000). This instrument cancels the flux of the quiet-Sun component by correlating the signals from two small antennas, whereas full sensitivity is achieved towards a burst originating from a region much smaller than the whole Sun. Thus flux density fluctuations caused by atmospheric modulation of the quiet-Sun component are reduced to a 
large extent. This results in an strongly improved signal level helpful to identify smaller bursts. Uncertainties of the absolute flux density are estimated at $25 \%$ and are dominated by the unknown flare position and the uncertainty of the quiet-Sun temperature. Atmospheric conditions at Bern University were good on 25 August 2001, with an estimated zenital opacity of 0.2 nepers measured at 16:00 UT and 17:00 UT before and after the flare. The presence of clouds has also been checked using an optical sensor, whose signal did not show any temporal variations during the studied solar flare.

We also used $\mu$-wave total flux measurements obtained by the Owens Valley Solar Array (OVSA; Gary and Hurford, 1999) at 40 frequencies in the 1-18 GHz frequency range, with $8 \mathrm{~s}$ time resolution, as well as full disk radio fluxes provided by the Radio Solar Telescope Network (RSTN) obtained up to $15.4 \mathrm{GHz}$ with $1 \mathrm{~s}$ time resolution.

Soft and hard X-ray, and $\gamma$-ray data were available from the different instruments (Soft and Hard X-ray telescopes (SXT, HXT), Wide Band Spectrometer (WBS)) onboard the Yohkoh satellite (Tsuneta et al., 1991; Kosugi et al., 1991; Yoshimori et al., 1991). These data were calibrated and analyzed using SolarSoft procedures.

\subsection{THE FLARE OVERALL TIME PROFILE}

Figure 1 describes the flare time history at, from top to bottom, 212, 405, 89.4, and $15.4 \mathrm{GHz}$, and in $53-93 \mathrm{keV}$ and $5.6-30 \mathrm{MeV}$ photon energy ranges. Units are $\mathrm{SFU}\left(1 \mathrm{SFU}=10^{-22} \mathrm{~W} \mathrm{~m}^{2} \mathrm{~Hz}\right)$ for millimeter and microwave time profiles, and counts $\mathrm{s}^{-1}$ for hard $\mathrm{X}$ ray (HXR) and $\gamma$-ray light curves. The submillimeter time profiles are in antenna temperatures (Kelvin), corrected for atmospheric opacity, and were obtained by beam $3(212 \mathrm{GHz})$ and beam $5(405 \mathrm{GHz})$. The reason not to use flux densities is that during most of the time period 16:31:13-16:32:10 UT at least one of the three SST $212 \mathrm{GHz}$ channels saturated, thus preventing the source absolute flux density determination, as burst source location computation could not be performed. At 16:31:13 UT the computed flux densities were of $10900 \pm 2400 \mathrm{SFU}$ and $5500 \pm 2200 \mathrm{SFU}$ at $212 \mathrm{GHz}$ and $405 \mathrm{GHz}$, respectively. At this time the source was located between the solar projection of beams 3 and 5, implying that any in-phase variations of the signals detected by these two beams actually reflect similar variations of the emitting source total flux density, rather than changes of its relative (to beams') position for which we would expect anticorrelated signals (Makhmutov et al., 1998).

Based on the following remarks, we have divided the whole time profile in four distinct time periods, (I), (II), (III) and (IV):

(I) 16:28-16:29:10 UT: During (I) only faint $\mu$-wave emission is detected. No millimeter, submillimeter or X-ray emissions are detected during this time interval. 
(II) 16:29:10-16:30:45 UT: This time period (II) corresponds to the first, relatively gradual increase of the emission at all radio frequencies and all photon energies except for gamma-rays.

(III) 16:30:45-16:31:05 UT: At 16:30:45 UT we note a sharp increase of the flare emission at all radio frequencies. The time period (III) also corresponds to the first appearance of HXR and $\gamma$-ray emission up to few MeVs photon energies. Figure 1 shows that during (III) common time structured emission is observed in all energy bands.

(IV) 16:31:05-16:32:30 UT: At 16:31:05 UT begins the time period (IV) which constitutes the main energetic part of the observed flare. Figure 1 shows that 89.4, 212, and $405 \mathrm{GHz}$ emissions increase dramatically. Similar increases with common temporal fine structures are observed, in particular for microwave emission and hard X-ray $/ \gamma$-ray count rates. The time period (IV) ends when counts are no longer detected above $5.6 \mathrm{MeV}$.

In Figure 2 we show a detailed time history of period (IV). We note a good correspondence between the submillimeter time profiles and the count rate history at 53-93 keV. In particular the former time curves exhibit four clear structures (denoted a, b, c, and d, shown using dashed lines) of roughly 5-20 s each, which are also seen in the HXR curve. On the other hand, the millimeter time profile at $89.4 \mathrm{GHz}$ only shows the first (a) and the last (d) of the four surges. During the period 16:31:20-16:31:43 UT (peaks $b$ and c), when the HXR and the $\gamma$-ray light curves increase toward their maximum, the millimeter emission at $89.4 \mathrm{GHz}$ remains partly steady. We also note that, although we cannot formally compute the 212 and $405 \mathrm{GHz}$ flux densities during the time period 16:31:14-16:32:09 UT, the time evolution of the source flux density during this period will be similar to that of the observed antenna temperatures, since they clearly present similar time profiles (see above explanations). This assumes that the emitting sources have the same angular size at 212 and $405 \mathrm{GHz}$, and that they are located at the same position. Owing to these conditions, the $212-405 \mathrm{GHz}$ flux index can be obtained from the 212-405 GHz radio antenna temperature index.

\subsection{TYPICAL OBSERVED SPECTRA DURING THE IMPULSIVE PHASE}

In Figure 3 we show examples of radio spectra observed during the impulsive phase of the flare. The spectra were obtained at 16:28:23 UT (no symbol) during the time period (I), at 16:29:50 UT (crosses) and 16:30:31 UT (stars) during the time period (II), at 16:31 UT (diamonds) during the time period (III), and at 16:31:13 UT (triangles) and 16:32:10 UT (squares) during the time period (IV). Figure 3, as well as a detailed analysis of the time evolution of the radio spectra, show that: (i) during (I) the microwave spectra are typical of non-thermal gyrosynchrotron emission with a spectral peak, $v_{\text {peak }}$, in the range $8-10 \mathrm{GHz}$ (Nita, Gary, and Lee, 2004). The optically thick part of the spectra have a slope, $\alpha_{\text {thick }}$ of about 2.0-2.2; (ii) during the next time period, (II), the spectral peak is slightly shifted towards 


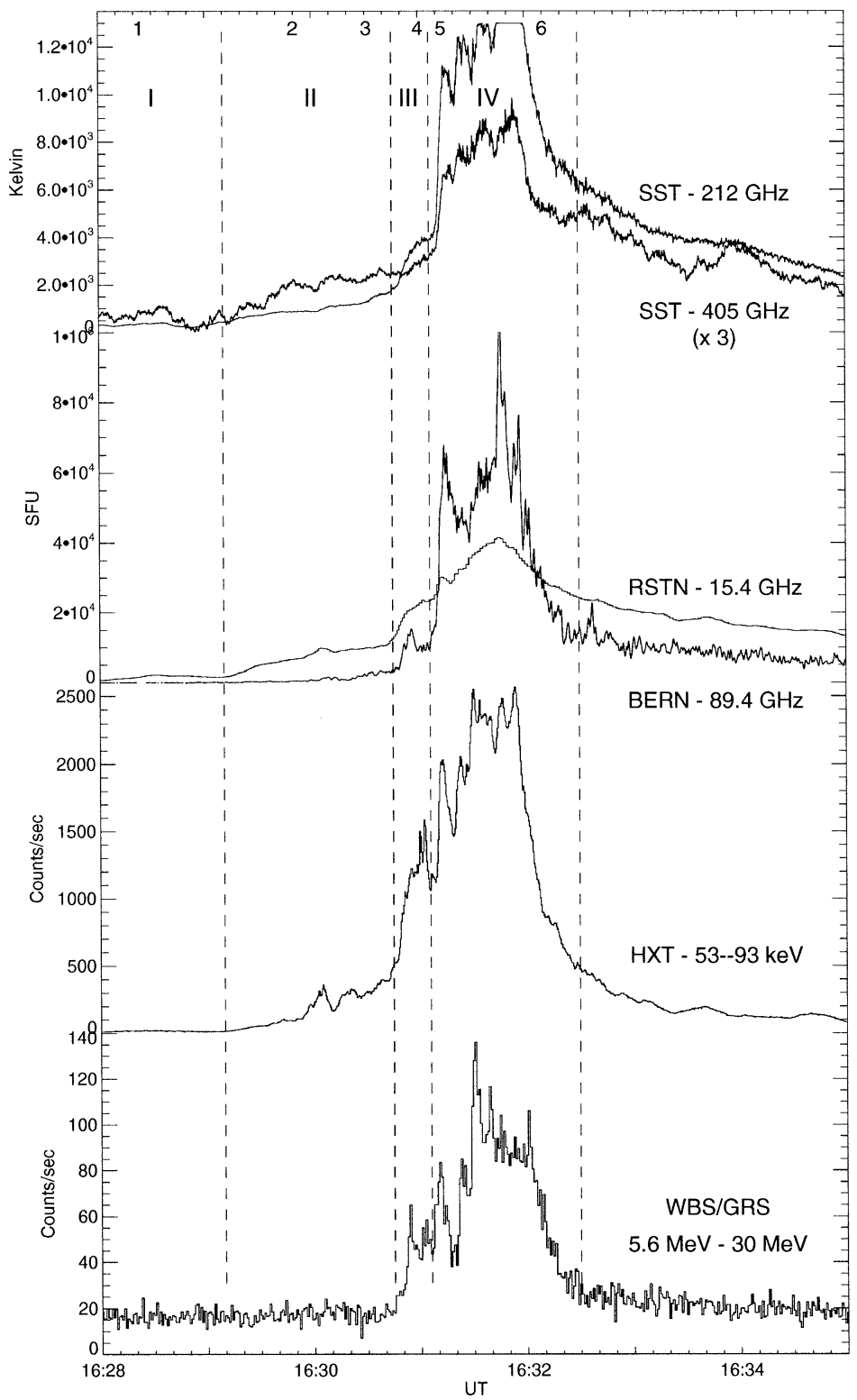

Figure 1. Multi-frequency flare time profile. From top to bottom: 405, $212 \mathrm{GHz}$ antenna temperatures (K); 89.4, 15.4 GHz total flux densities (SFU); hard X-ray count rate in the 53-93 keV channel; $\gamma$-ray count rate for energy above $5.6 \mathrm{MeV}$. The dashed vertical lines identify four different time periods noted I to IV. Numbers 1-6 on top indicate times of observed spectra shown in Figure 3. 


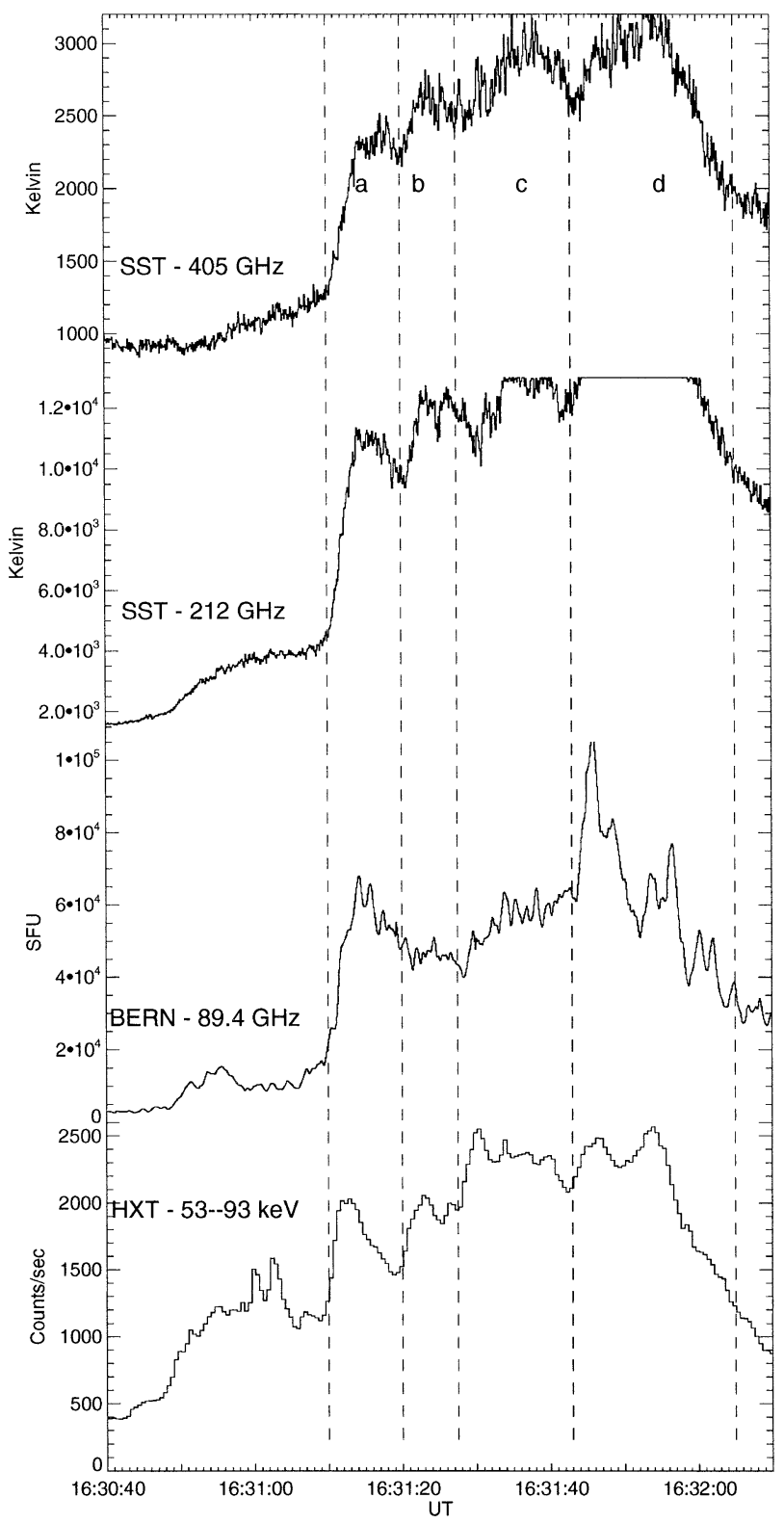

Figure 2. Similar to Figure 1 for the submillimeter, millimeter and hard X-ray emissions during the time period 16:30:40-16:32:10 UT. 


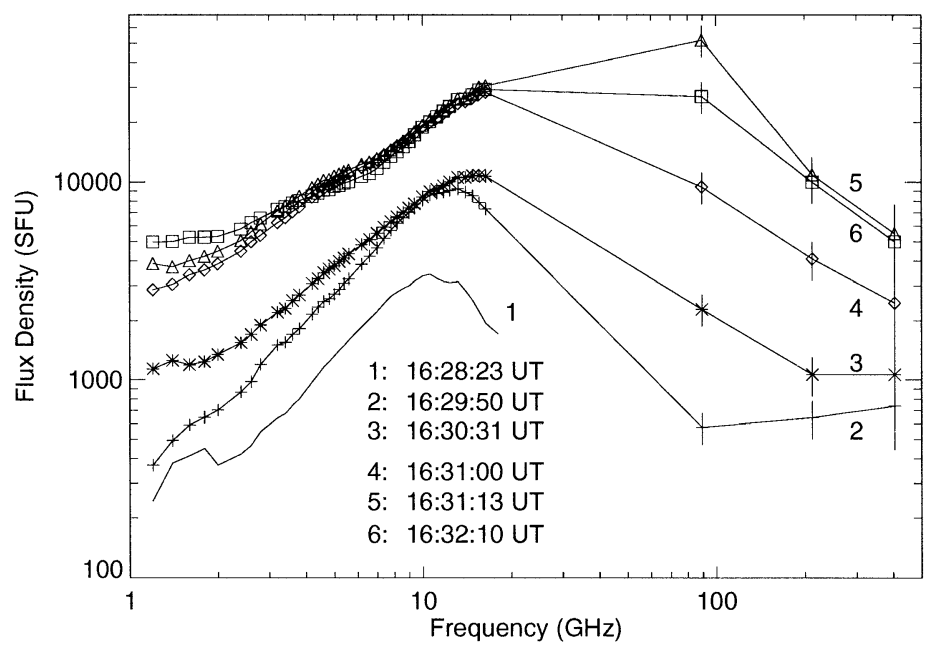

Figure 3. Observed radio spectra during the time period (I) (no symbol), the time period (II) (crosses and stars), the time period (III) (diamonds), the time period (IV) (squares and triangles).

higher frequencies at around $12-13 \mathrm{GHz}$, while $\alpha_{\text {thick }}$ gradually decreases down to about 1.2; (iii) during the time period (III) $v_{\text {peak }}$ is not observed any longer and lies between $18 \mathrm{GHz}$ and $89.4 \mathrm{GHz}$. As during the previous time period, $\alpha_{\text {thick }}$ decreases to reach a value of $0.7-0.9$; (iv) during the most intense part of the event, (IV), as during the time period (III), the spectral peak is not observed and the optically thick part of the spectra are rather flat at $\alpha_{\text {thick }} \sim 0.9$, as is common for X-class flares (Lee, Gary, and Zirin, 1994). We finally note that in the early stage of the flare, in particular during the time period (II), the sub-millimeter radio emission is not simply an extension of the microwave radio spectrum, but rather indicates that another (perhaps non-gyrosynchrotron) radiation process is dominant. Later on, during the main phases of the flare, the high frequency emissions are consistent with an extension of the microwave spectrum, suggesting that synchrotron emission is the dominant radiation mechanism throughout the range $1-405 \mathrm{GHz}$.

\subsection{Spatially RESOLVED X-RAY EMISSION FROM NOAA 9591}

In Figure 4 we summarize a detailed analysis of the time sequence of the spatially resolved soft X-ray (SXR, black contours) emitting plasma obtained by SXT. Frames in Figure 4 have been chosen with emphasis on noticeable changes of the SXR spatial distribution, which we compare with hard X-ray (HXR, white contours) $53-93 \mathrm{keV}$ images. Figure 4 elicits the following comments:

(1) In the pre-flare stage (16:27:19 UT) loop-like structures are observed connecting different sites of NOAA 9591 located on both sides of the main magnetic neutral line (NL) (thick dashed white line). Many of these are anchored in the penumbrae of the western (Wsp) and eastern (Esp) main spots seen in the background white-light image. 

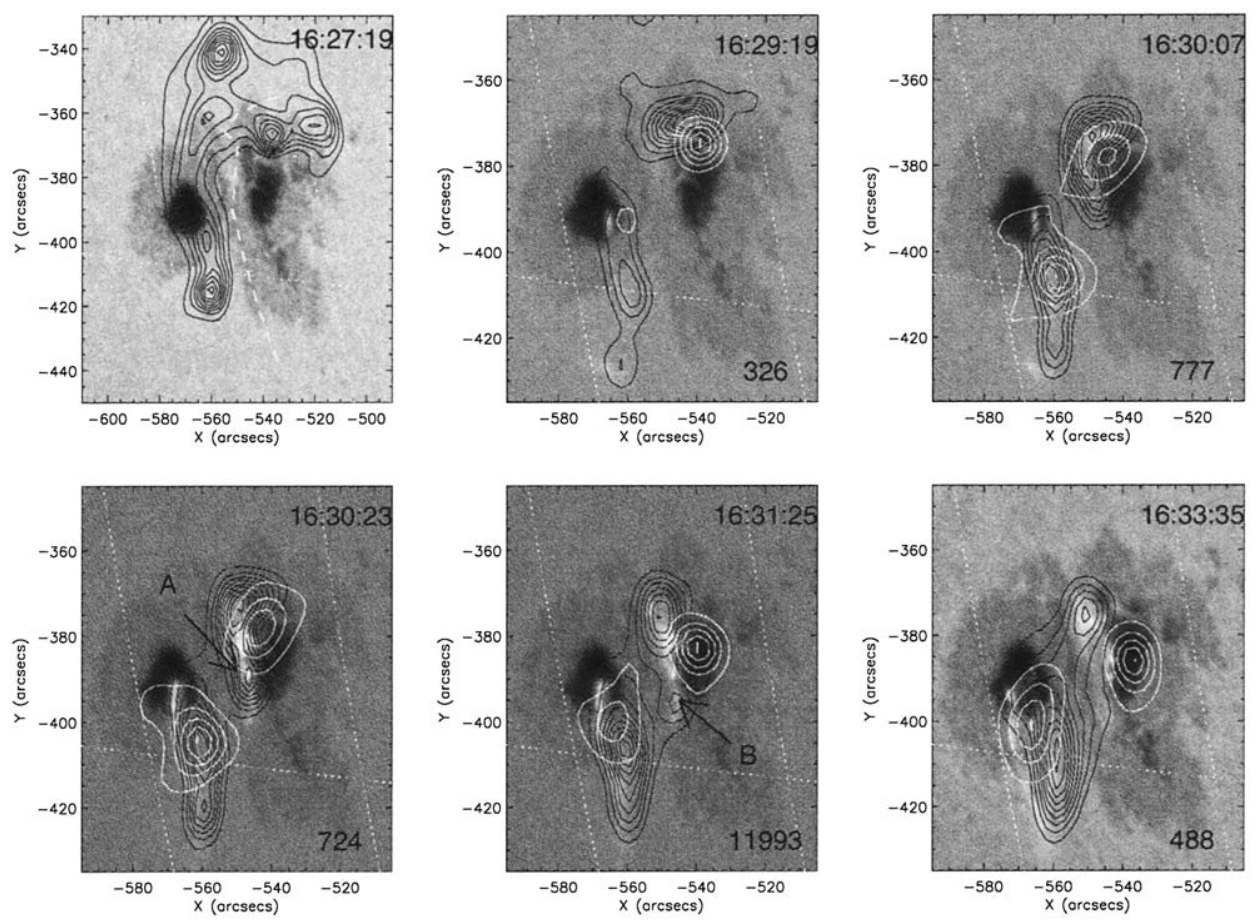

Figure 4. Time sequence analysis of SXT images (black contours) obtained with the A1 12 SXT filter. Levels are shown using $10 \%$ increasing steps from $15 \%$ up to to $95 \%$ of the maximum emission. White contours show 53-93 keV images obtained by accumulating counts during $10 \mathrm{~s}$, and where the levels are $20,40,60,80$, and $99 \%$ of the maximum emission indicated at the bottom right of each frame, in units of counts/sub-collimator. Contours are superimposed on the closest white-light image obtained by TRACE. The six panels have a field of view (FOV) of $1.5 \operatorname{arc~min}^{2}$ except for the first one (16:27:15 UT) for which the FOV is $2 \operatorname{arc~} \mathrm{min}^{2}$.

(2) At the beginning of time period (II) (16:29:19 UT), a bright SXR emission is observed north of Esp overlying the NL. This newly formed SXR source is observed to be co-spatial with a white-light brightening and, to a lesser extent, with a compact HXR source. On the other side of the NL another faint HXR source is detected close to the umbra of Esp.

(3) The next two frames, also obtained during time period (II), show the appearance of new and bright emissions in soft X-rays. They appear successively above and along the NL, giving the impression that the former source observed at 16:29:19 UT is gradually shifting its position in a southward direction. At 16:30:23 UT a new compact SXR source (denoted A) becomes spatially well separated from the main component emission. The apparent southern shift of the SXR emission is associated with a similar one seen in white light. In contrast to the frame at 16:29:19 UT, the eastern HXR emission is now brighter than that in the western part of the NL. 
(4) Starting from 16:30:40 UT, which corresponds to the beginning of time period (III) and to an increase of the flare emission at all frequencies from $\mu$-wave up to $\gamma$-ray emission (see Figure 1) a new compact ( $\sim 7$ arc sec) and bright SXR source is detected south of the previous source A, although at this time both are spatially unresolved. At 16:31:25 UT (during time period (IV)), this new source (denoted B) is brighter than the previous one (A). At this time the relative intensity of the east and west HXR emissions changed again, the west source being the brighter.

(5) At 16:33:35 UT, when the $\gamma$-ray emission vanished, the bright source B seems to have merged with the SXR emission from the eastern part of the magnetic neutral line, to form a more extended structure. Similarly, the northern SXR emission is now directly connected with the eastern part of the active region by a bright bridge which extends over the NL.

\subsection{SUMmARY OF OBSERVATION RESUlTS}

In summary, Figure 4 reveals that, as the event progresses in time, new SXR structures appear and participate in the flare development. They are bright, localized and compact (5-10 arc sec), suggesting low-lying small loops, or loop systems, merging in time with pre-existing structures, to form more extended emission sources extending up to 30 arc sec, which connect active region sites on both sides of the the magnetic inversion line. These new structures appear in connection with the development of the radio emission in the different time periods defined in Figure 1. In particular the appearance of source B was closely related in time to the onset of the most intense period of the flare impulsive phase.

\section{Results and Discussion}

\subsection{RADIO SPECTRUM DURING THE IMPULSIVE PHASE}

Figure 5 shows the $1-405 \mathrm{GHz}$ radio spectra observed during the time period IV (16:31:13 UT) and during the decreasing part of the event (16:32:10 UT). This figure and the earlier remarks made in Section 2.3 (see also Figure 3) indicate: (i) $v_{\text {peak }}$ increases from $\sim 7-10 \mathrm{GHz}$ to $\sim 12-13 \mathrm{GHz}$ during (I) and (II) and rises above $18 \mathrm{GHz}$ periods (III) and (IV); (ii) the optically thick slope of the spectrum gradually decreases down to $0.7-0.9$ (during (IV)), i.e., the observed optically thick spectra are much less steep than what is expected for self-absorption of gyrosynchrotron/synchrotron emission $\left(\alpha_{\text {thick }} \sim 2.5-2.9\right.$, Dulk, 1985). Suggesting a broad spectral peak in particular during (III) and (IV) when $v_{\text {peak }}$ lies somewhere between 18-70 GHz. These properties can be explained by an inhomogeneous emitting radio source (Lee, Gary, and Zirin, 1994), or the superposition of various emitting radio sources in different regions with different physical parameters 


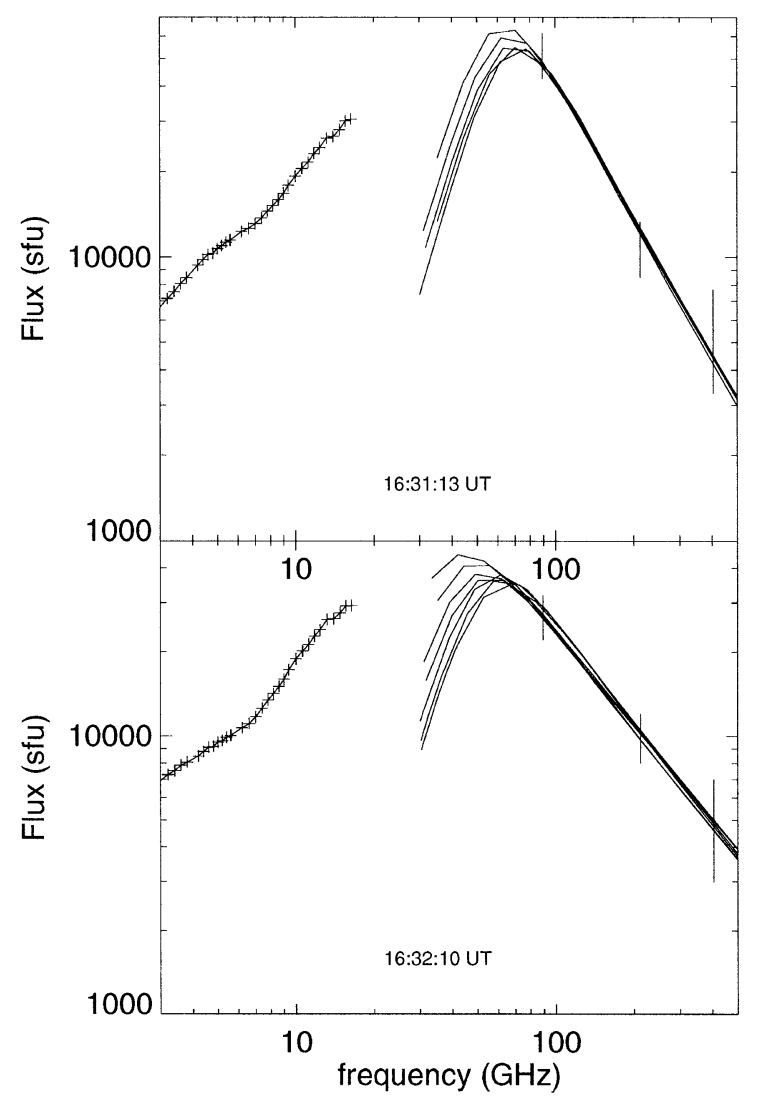

Figure 5. Observed 3-405 GHz radio flux spectra at 16:31:13 UT (top) and 16:32:10 UT (bottom). The data from 1-18 GHz are from OVSA, and the vertical bars are the SST flux densities and their range of uncertainty. The curves represent the theoretical synchrotron spectra for magnetic fields between 500-1100 G (top) and between 300-1500 G (bottom).

(Klein and Trottet, 1984; Klein and Magun, 1986). This interpretation is also supported by the time history of the flare as detected in soft X-rays, since we observed that consecutive compact sources appeared and merge together (or merge with preexisting sources), to form more extended structures. Therefore in the course of the flare, additional sites within the active region, with different physical conditions, participated in the production of energetic particles, resulting in the observed flat optically thick radio spectra. This interpretation is also in agreement with previous multi-frequency results obtained during smaller flares (Raulin et al., 2000), by comparing millimeter radio emission with the dynamics of active region magnetic field structures as traced by imaging soft $\mathrm{X}$-ray emissions.

Figure 1 shows a very high level of millimeter and submillimeter activity, which we used to define the time periods (III) and (IV) as the fast, impulsive phase of the event. The millimeter/submillimeter emissions are strong indications of the presence of very-high-energy electrons, since such emissions can only be generated 


\section{TABLE I}

Results obtained from the fits at 16:31:13 and 16:32:10 UT, showing the input model parameters (magnetic field and energy electron index $\delta$ ), as well as derived quantities $N_{>20 \mathrm{keV}}$, $N_{>1 \mathrm{MeV}}, E_{>20 \mathrm{keV}}$, and $E_{>1 \mathrm{MeV}}$.

\begin{tabular}{lllllll}
\hline $\begin{array}{l}\text { Time } \\
(\mathrm{UT})\end{array}$ & $\begin{array}{l}B \\
\text { (Gauss) }\end{array}$ & $\delta$ & $\begin{array}{l}N_{>20 \mathrm{keV}} \\
\left(10^{36} \mathrm{el} .\right)\end{array}$ & $\begin{array}{l}N_{>1 \mathrm{MeV}} \\
\left(10^{32} \mathrm{el} .\right)\end{array}$ & $\begin{array}{l}E_{>20 \mathrm{keV}} \\
\left(10^{29} \mathrm{ergs}\right)\end{array}$ & $\begin{array}{l}E_{>1 \mathrm{MeV}} \\
\left(10^{25} \mathrm{ergs}\right)\end{array}$ \\
\hline $16: 31: 13$ & $500-1100$ & $3.7-3.9$ & $175-8.6$ & $20.7-2.2$ & $85-4.4$ & $505-56$ \\
$16: 32: 10$ & $300-1500$ & $2.9-3.0$ & $4.4-0.089$ & $17.5-0.53$ & $2.8-0.06$ & $555-17.5$ \\
\hline
\end{tabular}

at high harmonics $s$ of the gyrofrequency and only relativistic/ultra-relativistic particles can radiate at such high $s$. Properties of these particles can be inferred by studying the optically thin part $(\tau \ll 1)$ of the observed spectra. For this we computed the expected thin synchrotron flux radiated by non-thermal electrons in a homogeneous magnetic field, in order to reproduce the observed fluxes. As mentioned in the introduction, we used the exact code developed by Ramaty (1969), Ramaty et al. (1994), with a power-law distribution (spectral index $\delta$ ) of nonthermal electrons with energy in the range $0.01-100 \mathrm{MeV}$ and a viewing angle of 45 deg. Although it does not affect the high frequency $\left(v>v_{\max }\right)$ part of the spectrum, we have chosen an equal value for the plasma and the gyro-frequencies. The calculations have been made for homogeneous sources with different magnetic field strengths (between 300 and $1500 \mathrm{G}$ ) and injected electron energy index $\delta$. We have fitted the optically thin part of the observed spectra at 16:31:13 UT during the early impulsive phase, and at 16:32:10 UT after the impulsive phase. The results are shown in Figure 5, superimposed on the observed spectra. We do not attempt to match the optically thick part of the spectrum. As mentioned above, the observed spectra are much flatter due to the significant inhomogeneity, which we did not include in our model. At 16:31:13 UT (top) the radio spectrum is steep, and the fit was obtained using magnetic fields values between 500 and $1100 \mathrm{G}$. We note that fits obtained using magnetic field higher than $1100 \mathrm{G}$ should be excluded since they produce a peak frequency at or above $90 \mathrm{GHz}$, which was not observed. At 16:32:10 UT the data seem to be well represented by the synchrotron emission of energetic electrons in a magnetic field of $\geq 300 \mathrm{G}$ with a spectral energy index $\delta \sim 2.9-3$.

The input model parameters (magnetic field, electron index $\delta$ ) as well as the obtained results are shown in Table I, where we indicate the instantaneous number of electrons above $20 \mathrm{keV}\left(N_{>20 \mathrm{keV}}\right)$ and above $1 \mathrm{MeV}\left(N_{>1 \mathrm{MeV}}\right)$ needed to explain the observed radio fluxes in the optically thin part of the radio spectra. The corresponding instantaneous electron energy contents $\left(E_{>20 \mathrm{keV}}\right.$ and $\left.E_{>1 \mathrm{MeV}}\right)$ are also shown in Table I. 


\subsection{INSTANTANEOUS MM/SUBMM-WAVE EMITTING ELECTRONS AND THE RELATION BETWEEN BOTH EMISSIONS}

The interest of studying high frequency radio flare observations lies in the fact that they are due to high energy electrons. For example in order to radiate at $405 \mathrm{GHz}$ some of these electrons need to have been accelerated up to $9-14 \mathrm{MeV}$ for an assumed magnetic field in the range 500-1100 G. Bremsstrahlung emission from electrons at these energies can contribute to the observed gamma-ray flux above $5.6 \mathrm{MeV}$.

The radio spectrum at 16:31:13 UT (Figure 5 top) during the impulsive phase has been found to agree with the synchrotron emission of injected electrons with a power-law energy distribution with $\delta=3.7-3.9$. As said earlier, the magnetic field in the emitting region should be at this time $\leq 1100 \mathrm{G}$ to ensure that the $89.4 \mathrm{GHz}$ emission is optically thin. On the other hand the flux of radio emitting electrons at say, $200 \mathrm{keV}, F_{R}^{200}$, obtained by extrapolating the radio electron spectrum to lower energies, must be lower than that of the X-ray emitting electrons, $F_{X}^{200}$. Otherwise radio emitting electrons should be responsible for the hard X-ray emission detected in the HXT channels, implying an X-ray electron emitting energy distribution with a power-law shape and an index of 3.7-3.9, contrary to what is observed (Metcalf et al., 2003). $F_{X}^{200}$ is estimated to be $5 \times 10^{33} \mathrm{e}^{-} \mathrm{s}^{-1}$ in agreement with the values obtained by Metcalf et al. (2003). $F_{R}^{200}$ can be evaluated as $F_{R}^{200} \sim \frac{N_{>200 k \mathrm{kV}}}{T}$ where $N_{>200 \mathrm{keV}}$ is computed at $200 \mathrm{keV}$, similar to what was done in Table I. For directly precipitating electrons, $T$ is the travel time of, or similarly the time spent by, the $200 \mathrm{keV}$ electrons in a $\sim 10$ arc sec region, and does not exceed a few tens of ms. Consequently only for high magnetic field values $(B \geq 1000 \mathrm{G})$ will the relation $F_{R}^{200} \leq F_{X}^{200}$ be satisfied. We are thus led to the finding that the magnetic field in the radio source emitting region is quite high, $\sim 1000-1100 \mathrm{G}$.

We do not have the size of the radio emitting region; but if we infer it from the size of the hard X-ray footpoints of $\sim 10$ arc sec which gives a volume of $\sim 9.0 \times$ $10^{25} \mathrm{~cm}^{-3}$, we get instantaneous density numbers of $n_{>20 \mathrm{keV}} \sim 1.2 \times 10^{11} \mathrm{~cm}^{-3}$ and $n_{>1 \mathrm{MeV}} \sim 3.1 \times 10^{6} \mathrm{~cm}^{-3}$, above $20 \mathrm{keV}$ and above $1 \mathrm{MeV}$, respectively, assuming as found earlier a magnetic field of $1000 \mathrm{G}$. The density $n_{>20 \mathrm{keV}}$ corresponds to an energy density of $\sim 6100 \mathrm{erg} \mathrm{cm}^{-3}$, which is the entire magnetic energy available in a volume of $1 \mathrm{~cm}^{-3}$ with a field strength of $\sim 390 \mathrm{G}$. If the energy in nonthermal electrons is to be converted from free, non-potential magnetic energy. i.e., about a few percent of the magnetic energy (Parker, 1983), it therefore strengthens the need for a high (above $1 \mathrm{kG}$ ) magnetic field strength in the source region.

During the more intense part of the event (16:31:20-16:31:40 UT, peaks $b$ and c in Figure 2) the above numbers are likely to increase. Metcalf et al. (2003) have deduced from HXT data that the shape of the electron injection spectrum stayed unchanged through the period 16:31:20-16:32 UT, and that the power in $>20 \mathrm{keV}$ electrons increased by a factor $\sim 1.4$. Therefore the increase of the hard $\mathrm{X}$-ray emission (see Figures 1 and 2) indicates a similar increase of the number 
of accelerated electrons above $20 \mathrm{keV}$. We then expect during the peaks $\mathrm{b}$ and $\mathrm{c}$ non-thermal electron densities above $20 \mathrm{keV} n_{>20 \mathrm{keV}} \geq 1.7 \times 10^{11} \mathrm{~cm}^{-3}$. Such high densities will in turn affect the radio emission through the synchrotron selfabsorption process (Klein, 1987), in which the synchrotron emission is reduced by the population of the radiating electrons itself. Assuming that the $212 \mathrm{GHz}$ source position is stable during peaks a, b and c, the $212-405 \mathrm{GHz}$ radio flux index, $\alpha$, during peaks b and c, can be inferred from the $212-405 \mathrm{GHz}$ radio antenna temperature index (see comments in Section 2.2), and we find a $\alpha \sim-1.2$, leading to an electron energy index $\delta \sim 3.4$. Since for magnetic fields in the range 900$1100 \mathrm{G}$ the radio frequency of $89.4 \mathrm{GHz}$ is emitted at harmonic numbers of about 30, we are in the domain of validity for Dulk's approximations (Dulk, 1985). Thus we can compute the synchrotron emission peak frequency $v_{\text {peak }}$ (Equation (43) in Dulk, 1985) due to an injected electron distribution with an energy index $\delta \sim 3.4$ and a non-thermal electron density above $20 \mathrm{keV}$ of $n_{>20 \mathrm{keV}} \sim 1.7 \times 10^{11} \mathrm{~cm}^{-3}$. The obtained peak frequencies are in the range $105-115 \mathrm{GHz}$, thus implying that the flux density at $89.4 \mathrm{GHz}$ will be partly absorbed during peaks $\mathrm{c}$ and $\mathrm{d}$. Then the combination of a high magnetic field region $(\sim 1000-1100 \mathrm{G})$ and a high density of non-thermal electrons might explain the steady flat flux density profile at 89.4 GHz during peaks b and c, as shown in Figure 2, and why these structures do not show up at millimeter wavelengths as they do at higher frequencies and in hard X-rays.

The numbers found in Table I have to be compared with those obtained by Ramaty et al. (1994) who found $10^{34}$ electrons with energy greater than $1 \mathrm{MeV}$ in order to explain the millimeter emission during the 4 June 1991 solar flare. However in this case the assumed magnetic field was rather low $(300 \mathrm{G})$ and the flux density at $80 \mathrm{GHz}$ was very high $(\sim 140000 \mathrm{SFU})$, thus explaining the huge number of instantaneous electrons with energy above $1 \mathrm{MeV}$. But it should also be noted that the instantaneous number of electrons obtained can be substantially reduced if the particles accumulate in the corona, due to an efficient trapping for example. Similarly Trottet et al. (2002) found that $\sim 9.0 \times 10^{30}$ and $\sim 2.4 \times 10^{30}$ electrons above $1 \mathrm{MeV}$, radiating in a field of 300 and $600 \mathrm{G}$, respectively, are necessary to explain the high-frequency radio emission detected during a X1.1 class solar flare on 22 March 2000. However no good hard X-ray observations were available to constrain a lower magnetic field strength in the radio emitting region, contrary to the event studied here.

The flux of $\geq 20 \mathrm{keV}$ accelerated electrons for the 25 August 2001 is in the range $4-5 \times 10^{36}$ particles per second, for a magnetic field in the range 1000$1100 \mathrm{G}$. This is commonly observed during large X-class flares, and leads to about $10^{39}$ electrons accelerated over the entire flare duration. We mention that although the obtained number of energetic electrons is high, it is still one to two orders of magnitude below that inferred during 'giant flares', where $10^{39}$ electrons above $20 \mathrm{keV}$ are accelerated per second for a total number above $10^{41}$ electrons (Kane, Hurley, and McTiernan, 1995). Since the numbers deduced for the present flare are 
lower limits, it means that the energy requirements for the 25 August 2001 flare are high, implying a highly efficient acceleration mechanism able to produce $\geq 4 \times$ $10^{36}$ electrons/s above $20 \mathrm{keV}$ and $\geq 1.1 \times 10^{32}$ electrons/s above $1 \mathrm{MeV}$, acting in a dense medium embedded in a strong magnetic field. Recently White et al. (2003) studied the 23 July 2002 X4.5 flare and found also that a few $10^{10} \mathrm{~cm}^{-3}$ electrons above $20 \mathrm{keV}$ were needed to explain simultaneous hard X-ray and millimeter (35 and $80 \mathrm{GHz}$ ) observations. Even higher numbers are probably needed in this event since it was shown that the $35 \mathrm{GHz}$ emission was not optically thin. We finally note that in these examples, as in the event studied here $\left(n_{>20 \mathrm{keV}} \geq 10^{11} \mathrm{~cm}^{-3}\right)$, the pressure of non-thermal particles may be comparable to that of the core plasma pressure, for any plausible plasma density $\left(n_{e} \leq 10^{12} \mathrm{~cm}^{-3}\right)$; see also Hudson $e t$ al. (2001).

\subsection{DyNAMiCS OF THE ACTIVE REGION MAGNETIC FIELD STRUCTURES}

The observational results of Section 2.3 have shown that the behaviour of the magnetic field structures of AR 9591, as traced by SXT images, is quite dynamic since it involves the successive appearance of new bright and compact sources. As the flare progresses through different phases, each characterized by an increase of the number of accelerated particles (as shown by the time profiles in Figures 1 and 2), these sources link to form more extended (>30 arc sec) structures. This behaviour as already been reported using multi-frequency flare analysis (see, e.g., Raulin et al., 2000).

Of particular interest is the finding of a close relation in time between the merging of two compact magnetic systems (A and B) and the production of high energy electrons responsible for intense millimeter, sub-millimeter and $\gamma$-ray emissions. A suggestion is that the interaction of structures $\mathrm{A}$ and $\mathrm{B}$, both with each other and with the overlying magnetic field (since $\mathrm{A}$ and $\mathrm{B}$ were not observed before 16:30 UT), produces a rearrangement of the magnetic field in the active region, along with reconnection processes leading to the production of $\mathrm{MeV}$ electrons. Sources (A, B) are most of the time unresolved in the SXT images (perhaps as a result of the interaction). Figure 4 shows, e.g., two times for which A and B can be well separated. To test the above suggestion, we compute the temporal variations of the relative soft X-ray intensity between sources $\mathrm{A}$ and $\mathrm{B}$, i.e., the difference $I(t)=I_{\mathrm{B}}(t)-I_{\mathrm{A}}(t)$, from SXT maps. The result is shown in Figure 6 and compared with the WBS/GRS count rate above $0.8 \mathrm{MeV}$. We can see that both time profiles are closely related, which suggests that (i) the appearance of the compact source B and (ii) its interaction with source A played an important role in the production of high energy electrons at the beginning of the impulsive phase. A direct link between the temporal behaviour of compact magnetic systems $\mathrm{A}$ and $\mathrm{B}$ and the acceleration of particles to high energies leads us to identify their interaction region as the acceleration site, thus located at low altitudes of about 


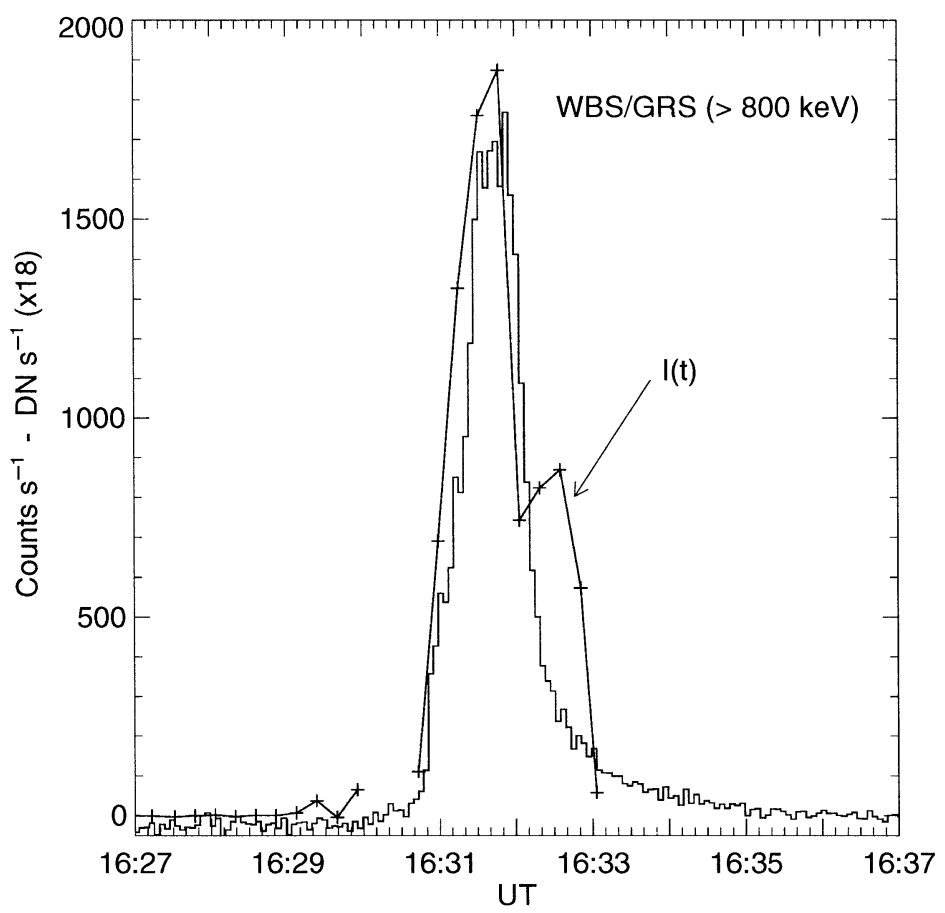

Figure 6. Relative SXR intensity, $I(t)=I_{\mathrm{B}}(t) / I_{\mathrm{A}}(t)$ of compact sources A and B (see Figure 4), obtained from Al12 filter images, and compared with the count rate detected above $0.8 \mathrm{MeV}$ by WBS/GRS (histogram).

$3000-5000 \mathrm{~km}$, if we assume the height of the compact magnetic structures is to be roughly equal to their diameter seen in SXT maps.

Low-lying acceleration regions have already been suggested in the literature (Grechnev and Nakajima, 2002). In that work it was argued that strong nonthermal flare processes, producing electrons radiating synchrotron emission up to $34 \mathrm{GHz}$, were caused by an interaction between compact low-lying magnetic loops. Similarly, Hanaoka $(1994,1999)$ and Raulin et al. (2000) show that solar flares can be triggered by interacting magnetic loops, one of them being generally much smaller than the others. Hanaoka (1999), using a detailed temporal analysis, concluded that the delays between remote and main flare emissions in hard X-rays and microwaves were compatible with the transport of energetic electrons from the interaction region between low-lying and large magnetic loops. In the case of the event studied in this paper, a low-lying acceleration region is also required to account for the high densities of non-thermal electrons deduced from radio observations and is consistent with the high magnetic fields inferred. Whether a location in the low solar atmosphere for the loop-interaction/acceleration region is a necessary condition to produce $\mathrm{MeV}$ electrons, capable of radiating submillimeter and $\gamma$-ray emissions, is unclear and deserves further similar studies. 


\section{Conclusions}

We have presented observations of a X5.3 solar flare covering a wide range of radio frequencies from $1 \mathrm{GHz}$ up to $405 \mathrm{GHz}$, including imaging X-ray data and $\gamma$-ray count rate time profiles up to $6.5-100 \mathrm{MeV}$ photon energies. The main results are as follows:

- A strong non-thermal impulsive phase was detected up to $405 \mathrm{GHz}$. At the beginning of this phase, the observed data are compatible with $4-5 \times 10^{36}$ electrons accelerated $(>20 \mathrm{keV})$ per second, radiating synchrotron emission in a 1000-1100 G magnetic field. At the peak of the flare the energy requirements, in terms of accelerated electron number, are higher, and are able to produce the self-absorption of the millimeter emission.

- The strong non-thermal impulsive phase was initiated when low-lying $(3000-5000 \mathrm{~km})$ compact loop systems interact with each other and with the overlying magnetic field. It is suggested that the acceleration region is located in dense, high magnetic field environments, rather than in the middle/high corona.

The results presented here show the ability of a combination of multi-frequency observations over a wide frequency and photon energy range to provide a better description and understanding of physical processes at work during solar flares. For this, the knowledge about acceleration/radiation sites as well as transport processes are important clues. In principle, $\gamma$-rays and submillimeter diagnostics give two independent, but complementary, types of information on the emitting electrons. This is because the mechanisms that produce these emissions depend on the injected electron spectrum (non-thermal bremsstrahlung and synchrotron) and the local magnetic field strength (synchrotron). Therefore comparing the two diagnostics should inform us about the transport of accelerated particles. Because of this, many clues are expected from future observations of solar flares, in particular those that will combine SST submillimeter data with that of high-energy imaging spectroscopy obtained by RHESSI.

\section{Acknowledgements}

This research was partially supported by Brazilian agencies FAPESP (contracts 99/06126-7 and 01/00604-6), CNPq (contracts 300782/96-9 and 304822/89-2) and the Swiss National Science Foundation (Grant 200020-100167). HH would like to acknowledge grant NAS 5-98033. We are grateful for the support of CASLEO engineers and technicians at the observatory of El Leoncito. We thank Dr Nicole Vilmer for stimulating discussions and comments. We also thank Dr Andreas Magun for checking the $89.4 \mathrm{GHz}$ data against any atmospheric effect. The Yohkoh mission of ISAS was prepared and operated by an international collaboration of Japanese, US and UK scientists. We thank M. Yoshimori for the use of Yohkoh/GRS 
time profiles. OVSA is supported by NSF grant AST-0307670 to New Jersey Institute of Technology.

\section{References}

Aschwanden, M. J. and Benz, A. O.: 1997, Astrophys. J. 480, 825.

Aschwanden, M. J., Wills, M. J., Hudson, H. S., Kosugi, T., and Schwartz, R. A.: 1996, Astrophys. J. 468, 398.

Dulk, G. A. and Marsh, K. A.: 1982, Astrophys. J. 259, 350.

Dulk, G. A.: 1985, Ann. Rev. Astron. Astrophys. 23, 169.

Gary, D. E. and Hurford, G. J.: 1999, Proceedings of the Nobeyama Symposium, held in Kiyosato, Japan, Oct. 27-30, 1998 in T. S. Bastian, N. Gopalswamy and K. Shibasaki (Eds.), in NRO Report No. 479., 429.

Giménez de Castro, C. G., Raulin, J.-P., Makhmutov, V. S., Kaufmann, P., and Costa, J. E. R.: 1999, Astron. Astrophys. 140, 373.

Grechnev, V. V. and Nakajima, H.: 2002, Astrophys. J. 566, 539.

Hanaoka, Y.: 1994, Astrophys. J. 420, L37.

Hanaoka, Y.: 1999, Publ. Astron. Soc. Japan 51, 483.

Hudson, H. S., Kosugi, T., Nitta, N. V., and Shimojo, M.: 2001, Astrophys. J. 561, L211.

Kane, S. R., Hurley, K., and McTiernan, J. M.: 1995, Astrophys. J. 466, L47.

Kaufmann, P. et al.: 2001a, in J. T. Pinho, G. P. S. Cavalcante and A. H. G. Oliviera (eds.), Proc. SBMO/IEEE/ MTT-S International Microwave and Optoelectronics Conference, (Piscataway:IEEE), p. 439.

Kaufmann, P. et al.: 2001b, Astrophys. J. 548, L95.

Kaufmann, P. et al.: 2002, Astrophys. J. 574, 1059.

Kaufmann, P. et al.: 2004, Astrophys. J. 603, L121.

Klein, K.-L.: 1987, Astron. Astrophys. 183, 341.

Klein, K.-L. and Trottet, G.: 1984, Astron. Astrophys. 141, 67.

Klein, K.-L., Trottet, G., and Magun, A.: 1986, Solar Phys. 104, 243.

Kosugi, T. et al.: 1991, Solar Phys. 136, 17.

Kundu, M. R., Grechnev, V. V., Garaimov, V. I., and White, S. M.: 2001, Astrophys. J. 563, 389.

Lee, J. W., Gary, D. E., and Zirin, H.: 1994, Solar Phys. 152, 409.

Lüthi, T. 1999, Master's thesis, Institute of Applied Physics, University of Bern, http://www.iapmw.unibe.ch/publications/pdfliles/139.pdf (in German).

Makhmutov, V. S., Costa, J. E. R., Raulin, J.-P., Kaufmann, P., Lagrotta, P. R., Giménez de Castro, C. G., Magun, A., and Arzner, K.: 1998, Solar Phys. 178, 393.

Makhmutov, V. S., Raulin, J.-P., Giménez de Castro, C. G., Kaufmann, P., and Correia, E.: 2003, Solar Phys. 218, 211.

Metcalf, T. R., Alexander, D., Hudson, H. S., and Longcope, D. W.: 2003, Astrophys. J. 595, 483.

Nita, G. M., Gary, D. E., and Lee, J.: 2004, Astrophys. J. 605, 528.

Parker, E. N.: 1983, Astrophys. J. 264, 642.

Ramaty, R.: 1969, Astrophys. J. 158, 753.

Ramaty, R., Schwartz, R. A., Enome, S., and Nakajima, H.: 1994, Astrophys. J. 436, 941.

Raulin, J.-P., Vilmer, N., Trottet, G., Nitta, N., Silva, A. V. R., Kaufmann, P., Correia, E., and Magun, A.: 2000, Astron. Astrophys. 355, 355.

Raulin, J.-P., Kaufmann, P., Giménez de Castro, C. G., Pacini, A., Makhmutov, V. S., Levato, H., and Rovira, M.: 2003, Astrophys. J. 592, 580.

Trottet, G., Raulin, J.-P., Kaufmann, P., Siarkowski, M., Klein, K.-L., and Gary, D. E.: 2002, Astron. Astrophys. 381, 694. 
Tsuneta, S. et al.: 1991, Solar Phys. 136, 37.

White, S. M. and Kundu, M. R.: 1992, Solar Phys. 141, 347.

White, S. M., Krucker, S., Shibasaki, K., Yokoyama, T., Shimojo, M., and Kundu, M. R.: 2003, Astrophys. J. 595, L111.

Yoshimori, M. et al.: 1991, Solar Phys. 136, 69. 